\title{
ATOM PROBE TOMOGRAPHY ANALYSIS OF POSSIBLE RHENIUM CLUSTERING IN NICKEL-BASED SUPERALLOYS
}

\author{
A. Mottura ${ }^{1}$, M.K. Miller ${ }^{2}$, R.C. Reed ${ }^{3}$ \\ ${ }^{1}$ Department of Materials, Imperial College London, London SW7 2BP, UK \\ ${ }^{2}$ Materials Science and Technology Division, Oak Ridge National Laboratory, Oak Ridge, TN 37831-6136, USA \\ ${ }^{3}$ Department of Metallurgy and Materials, The University of Birmingham, Edgbaston, Birmingham B15 2TT, UK
}

Keywords: Rhenium, APT, Clustering

\begin{abstract}
A local electrode atom probe is used to characterise a number of binary and commercial nickel-based alloys, specifically to determine whether rhenium atoms form solute clusters in these materials. The data are analysed via a statistical approach and results are compared to simulations which take the typical efficiency of the atom probe and the scatter in atomic positions into account. Thus, even the smallest clusters can - in principle - be detected by the algorithm. The analysis demonstrates conclusively that rhenium is not prone to clustering in the nickel lattice above the level associated with the random distribution of Re atoms. A similar conclusion applies to the other 5d elements ( Ta, W, Ir and Pt) analysed in this study.
\end{abstract}

\section{Introduction}

Rhenium is of great relevance to the superalloys: small additions of this element have been found to improve their creep properties considerably. Yet, the physical effects giving rise to this so-called rhenium-effect are not well understood and further fundamental work is required to clarify them [1]. It is widely accepted that an understanding of how rhenium is distributed in the nickel lattice will help explain the fundamental reasons behind the rhenium-effect.

In the past, several studies on Re in nickel-based superalloys have been performed with the use of the atom probe [2]. These studies began in the 1980s when Blavette et al. [3] used the one-dimensional (1D) atom probe to find some evidence of small $(1.3 \mathrm{~nm}$ diameter $)$ rhenium particles, by examination of ladder diagrams. Since this seminal work, rhenium clustering in these materials has been invoked as a possible explanation for the rhenium-effect. These studies were expanded by
Wanderka et al. [4] looking at the distribution of rhenium and other solute elements in nickel-based superalloys. Later studies by Warren et al. [5] and Miller et al. [6] focus on the rhenium enrichment at the $\gamma / \gamma^{\prime}$ interface, although no further study to explain this effect, and its ramifications, has been done. Yoon et al. [7] suggested that Re segregates at the $\gamma / \gamma^{\prime}$ interfaces and later studies focused on the effect of Re on secondary $\gamma^{\prime}$ precipitates and nanostructure evolution [8, 9]. The theory of rhenium clustering in nickel superalloys is presently suggested as the reason behind the rheniumeffect $[10,11]$. This theory is supported with a reference to the large miscibility gap in the $\mathrm{Ni}$-Re phase diagram and the low solubility of Re in the $f c c-\mathrm{Ni}$ lattice at room temperature.

More recently, the necessity for rhenium clustering to explain the rhenium-effect has been called into question $[12,13]$, since it has been pointed out that the rhenium/vacancy exchange energy in the nickel-rich matrix is remarkably high; thus rhenium is expected to retard all diffusion-driven processes in these materials, contributing to the retention of mechanical properties, particularly under creep conditions. Additionally, the solubility of $\mathrm{Re}$ in $\mathrm{Ni}$ at $500^{\circ} \mathrm{C}$ and above is high enough that the rhenium content of the $\gamma$ phase of a typical superalloy can be retained in solid solution. Furthermore, analysis performed by some of the authors with Extended X-ray Absorption Fine Structure [14], which probes the local atomic coordination of selected atoms, suggests that Re is not prone to solute clustering in the nickel-rich lattice [15]. These considerations indicate that there is uncertainty upon the atomic configuration of Re solute atoms in nickel-based superalloys. For this reason, no agreed mechanism for the rhenium-effect in these materials has yet been proposed. 
Atom probe technology has advanced significantly [16] since the pioneering work of Blavette and co-workers, so it was decided to characterise any possible rhenium clustering in these materials in a more detailed way than was possible in the 1980s. Extensive use is made of the latest atom probe tomography (APT) techniques, so that datasets of tens of millions of atoms can be collected resulting in a better statistical treatment of the data. Two distinct sets of specimens are examined. Binary nickel alloys $\mathrm{Ni}-\mathrm{X}$, where $\mathrm{X}$ is an element taken from the $5 \mathrm{~d}$ transition metal series, were analysed to observe the distribution of solute atoms when not affected by large microstructural features. Additionally, samples of a common single crystal superalloy of chemical complexity equivalent to those used commercially were also examined.

\section{Background}

In the atom probe, a high positive bias voltage is applied to a needle-shaped specimen. In order to field evaporate (i.e. remove) the surface atoms, either an additional short duration positive voltage pulse is superimposed on the standing voltage on the specimen or an equivalent negative pulse is applied to an annular counter electrode in front of the specimen. In the 1D atom probes used in the early studies, the resulting ions travel down the time-of-flight mass spectrometer to a single atom sensitive detector. In this variant, the ions collected originated from an area of the surface defined by an aperture approximately 1-2 $\mathrm{mm}$ in diameter positioned a few centimeters from the specimen. No information is obtained on the $x$ and $y$ positions of the ions within this small predefined area. In the Local Electrode Atom Probe (LEAP), the wide-angle position-sensitive detector is comprised of a pair of microchannel plates and a crossed delay line detector. This combination allows the $x$ and $y$ positions of the ions striking the single atom detector, and hence their original $x$ and $y$ coordinates in the sample, to be estimated [2]. In both types of atom probe, the $z$ coordinate is determined from the order in which the ions are collected, and the massto-charge-state ratio of the ion is estimated from the flight time, flight distance and the applied voltages [2]. From these data, a three-dimensional map of the atoms in the analyzed volume can be reconstructed in the LEAP.

Not all atoms present in the analyzed volume are detected. Typically, the detection efficiency of both the $1 \mathrm{D}$ and $3 \mathrm{D}$ versions of the atom probe is $\sim 50-60 \%$. The main loss of ions occurs when an ion strikes the interchannel region of the microchannel plate rather than the interior wall of the channel so that the ion-to-electron conversion and the subsequent electron multiplication process within the channel are not initiated. The open channel area of a microchannel plate is typically $62 \%$ of the surface area. Other minor loss mechanisms further decrease the detection efficiency [2].

Furthermore, the position of each detected atom can be displaced up to $\sim 5 \AA$ from its original location due to trajectory aberrations produced by the local environment of the evaporating atoms. For nickel these trajectory aberrations mean it is very hard to define lattice site occupation and crystal structure. In the 1D atom probe, a 1D string of atoms is collected in which adjacent atoms may not be nearest neighbour atoms in the sample due to field evaporation from different locations within the surface area that is exposed to the detector. Also due to the lower pulse repetition rate used in the $1 \mathrm{D}$ atom probe $(1-2 \mathrm{kHz}$ versus $200 \mathrm{kHz}$ in the LEAP), the 1D instrument is more prone to preferential evaporation of ions between the pulses and preferential retention of the refractory elements, such as Re, which may have an influence on the order of evaporation. These factors have a great impact on the ability to detect small clusters, especially when the size of these clusters approaches the typical positional scattering dimension.

Obviously, datasets of larger size should facilitate statistical analysis of greater accuracy. Nowadays, up to (and beyond) 100 million atoms can be collected from a given sample. This contrasts strongly with the situation for the original $1 \mathrm{D}$ atom probes used by Blavette [3], for which datasets of a few 1,000s of atoms were expected.

The analyses of Blavette [3] and Wanderka [4], reproduced in Figure 1, relied upon ladder diagrams obtained from 1DAP data. Ladder diagrams show the count of each species present in the sample over the total number of atoms collected. The overall slope of the line will yield the relative content of rhenium in the alloy (i.e. its atomic fraction). Variations in the slope correspond to perturbations in the distribution of rhenium atoms in the host lattice: these perturbations were suggested to be an indication of rhenium clustering in the alloy [3]. This type of analysis, however, needs to be applied to datasets which do not show compositional variations due to microstructural features. This is not true of the superalloys, where the two main phases $\left(\gamma\right.$ and $\left.\gamma^{\prime}\right)$ have very different rhenium concentrations. 

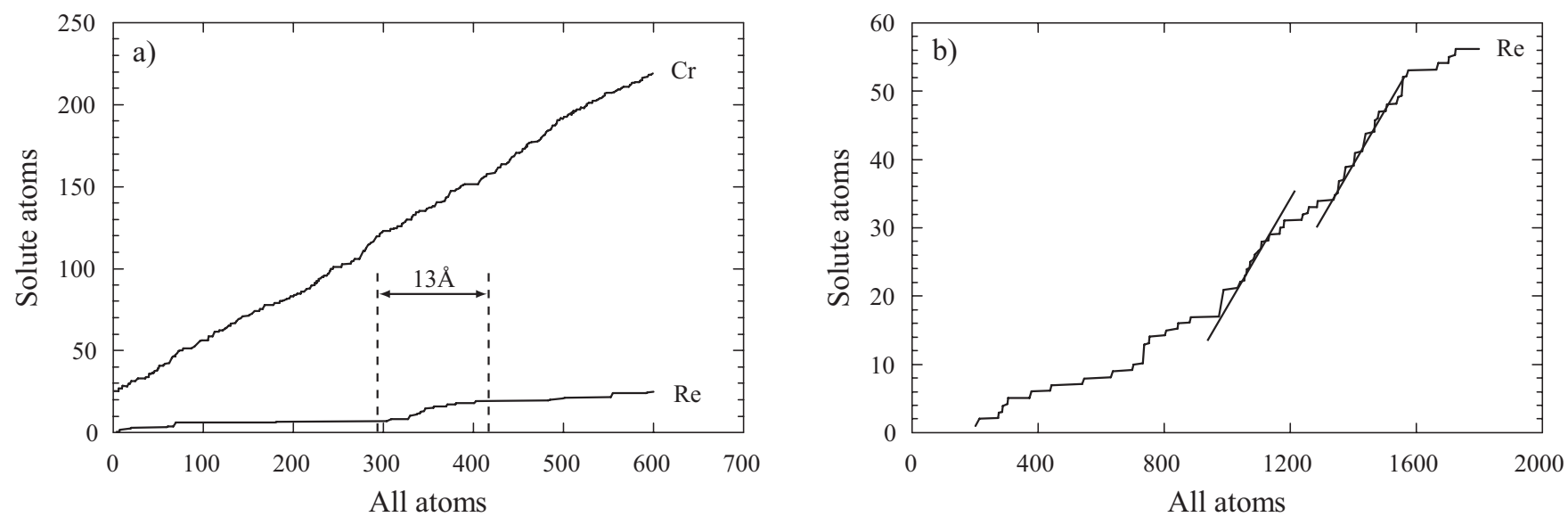

Figure 1: a) illustrates the discontinuities in the ladder diagram for a Re-doped PWA1480 superalloy in the early analysis by Blavette et al. $[3,10]$. b) illustrates the discontinuities in the ladder diagram for Re, as detected in CMSX-4 by Wanderka et al. [4].

In these early studies, these issues were addressed by examining samples taken solely from the $\gamma$ matrix phase, thereby eliminating the effect of the partitioning of rhenium from the $\gamma^{\prime}$ phase to the $\gamma$ phase as a source of distributional variation. Nevertheless, the distribution of rhenium in the $\gamma$ phase at room temperature can also be considered to be inhomogeneous: significant rhenium enrichment is found near the $\gamma / \gamma^{\prime}$ interfaces and small secondary $\gamma^{\prime}$ precipitates are found in the $\gamma$ matrix phase. Additionally, random fluctuations in composition are expected. Ladder diagrams can now be reproduced from the larger datasets obtained from modern atom probes, as shown in Figure 2. In order to obtain the ladder diagram, a narrow (user defined) square or round cross section cylinder is cut from the original three-dimensional volume: this can be done so that the cylindrical sample is made of $\gamma$ phase only and secondary $\gamma^{\prime}$ particles can also be avoided. Because of this careful sampling of the data, the rhenium distribution is more regular; nevertheless some fluctuations are still present (as indicated).

It follows from the above, that it is necessary to determine whether these fluctuations are due to solute clustering, or alternatively due to random variations in the distribution of the solute atoms in the lattice. At these solute concentrations, when the solute atoms are randomly distributed in the structure, small numbers of naturally occurring clusters are expected. In fact, for a 3 at.\% solute concentration only $80-90 \%$ of these solute atoms will be isolated in a single atom configuration (i.e. with no other solute nearest neighbours). The remaining $10-20 \%$ of the solute atoms will have one or more solute atoms as nearest neighbors, which can be considered

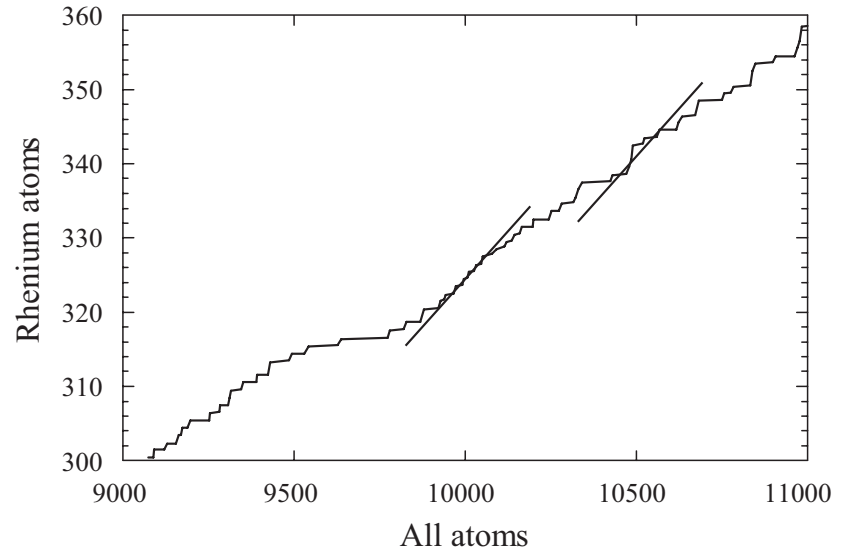

Figure 2: Illustration of how the discontinuous change in the slope of the Re ladder diagram can be reproduced by analysing data from a modern LEAP dataset. To reproduce the data of Blavette and Wanderka, this ladder diagram was generated by constructing a narrow cross section cylinder through 3D data acquired in a LEAP.

to be clusters. Both types of clusters will have an influence on the mechanical properties of the material. Ladder diagrams are not able to differentiate between the naturally occurring clusters from the additional solute clusters due to the limited statistics. In this paper, the terms rhenium cluster or solute cluster are reserved for the latter type and random cluster for the former type.

The research reported in this paper was performed to address this question. A large amount of data collected from a modern LEAP will be analysed. The results will be compared to datasets determined by numerical simulations to reinforce the conclusions drawn. 
Table I: The table shows the solute content of the binary alloys examined measured with the atom probe and the composition of CMSX-4 [17] in at.\%.

\begin{tabular}{ccccccccccccc}
\hline Elements & $\mathrm{Cr}$ & $\mathrm{Co}$ & $\mathrm{Mo}$ & $\mathrm{Al}$ & $\mathrm{Ti}$ & $\mathrm{Hf}$ & $\mathrm{Ta}$ & $\mathrm{W}$ & $\mathrm{Re}$ & $\mathrm{Ir}$ & $\mathrm{Pt}$ & $\mathrm{Ni}$ \\
\hline \hline CMSX-4 & 6.61 & 11.25 & 0.26 & 11.62 & 0.93 & 0.03 & 1.87 & 1.70 & 0.97 & - & - & Bal. \\
Ni-Ta & - & - & - & - & - & - & 3.70 & - & - & - & - & Bal. \\
Ni-W & - & - & - & - & - & - & - & 4.40 & - & - & - & Bal. \\
Ni-Re & - & - & - & - & - & - & - & - & 3.12 & - & - & Bal. \\
Ni-Ir & - & - & - & - & - & - & - & - & - & 3.40 & - & Bal. \\
Ni-Pt & - & - & - & - & - & - & - & - & - & - & 3.35 & Bal. \\
\hline
\end{tabular}

\section{Experimental and Analysis Details}

The binary alloys for atom probe tomography were prepared in the following way. A number of binary $\mathrm{Ni}-\mathrm{X}$ alloys were cast, where $\mathrm{X}$ was taken from the series of $5 \mathrm{~d}$ transition metals. Specifically, Ni-Ta, Ni-W, $\mathrm{Ni}-\mathrm{Re}, \mathrm{Ni}-\mathrm{Ir}$ and Ni-Pt alloys were chosen. These alloys were prepared in the form of $400 \mathrm{~g}$ ingots using vacuum induction melting. The composition of these alloys was chosen such that it would be comparable to the amount of rhenium in the matrix $\gamma$ phase of commercial alloy CMSX-4. To ensure homogenisation, annealing was performed at $1250^{\circ} \mathrm{C}$ for 7 days in flowing argon, and the material was allowed to furnace cool. According to the established $\mathrm{Ni}$ phase diagrams, the compositions chosen belong to the $f c c-\mathrm{Ni}$ phase from the annealing temperature to ambient [18]; thus solid solutions are expected with no precipitation reactions. The CMSX-4 samples were taken from the same set as an earlier study on rhenium in nickel superalloys by Miller and Reed [6]. Several millions of atoms were collected from each sample using the local electrode atom probe (LEAP) at Oak Ridge National Laboratory. The exact compositions of the binary alloys used and CMSX-4 are given in the Table I.

The clustering analysis was performed with a purposebuilt algorithm which explores the nearest neighbours of each solute atom in 3-dimensions and generates a count of clusters which are $n$-atoms in size. This means that the fraction of solute atoms in each cluster $n$-atoms in size can be determined. The nearest neighbours of a given atom are selected by looking at a distance $R_{\max }$ from the atom of interest. The amount and size of clusters detected will depend upon the value chosen for $\mathrm{R}_{\max }$. To make the analysis consistent, the value of $\mathrm{R}_{\max }$ was chosen to be half way between the first and second shell of neighbours, such that the average of detected nearest neighbours would be comparable to the expected number of nearest neighbours.

The results are compared to simulated AP datasets [19] which take into account the efficiency and positional scatter of the atoms comparable to that of the real datasets. The simulation software employed creates a grid of lattice sites with a determined crystal structure and lattice parameter. Spherical clusters of solute atoms (B) of a given size are distributed to match a set number density within the volume. The rest of the lattice sites (those not belonging to a cluster) is populated with solvent atoms (A) or solute atoms (B), with the presence of (B) atoms matching a given concentration. After this allocation of solute, a given percentage of the atoms are deleted at random to match the detection efficiency. The rest of the atoms will undergo a displacement from their perfect lattice site which follows a Gaussian distribution over a determined range in the 3 dimensions. All the simulations in this study were constructed on an underlying $f c c$ lattice with a lattice parameter of $3.52 \AA$. The efficiency was set at $60 \%$ for all simulations and the positional scattering was set to $\pm 5 \AA$.

The analysis on the simulated datasets was done to monitor the effect of scattering and efficiency on solute clusters of different sizes and distribution and to evaluate the performance of the algorithm used to detect clusters. This is important in order to obtain the lowest limit of cluster size and distribution which can be determined from the analysis.

\section{Results}

Analysis of simulated datasets

A difficulty for studying clusters using the atom probe is that the detection efficiency and position scattering might disperse the atoms which form clusters. This is particularly true when the size of the clusters is very small such that it approaches the typical atomic 

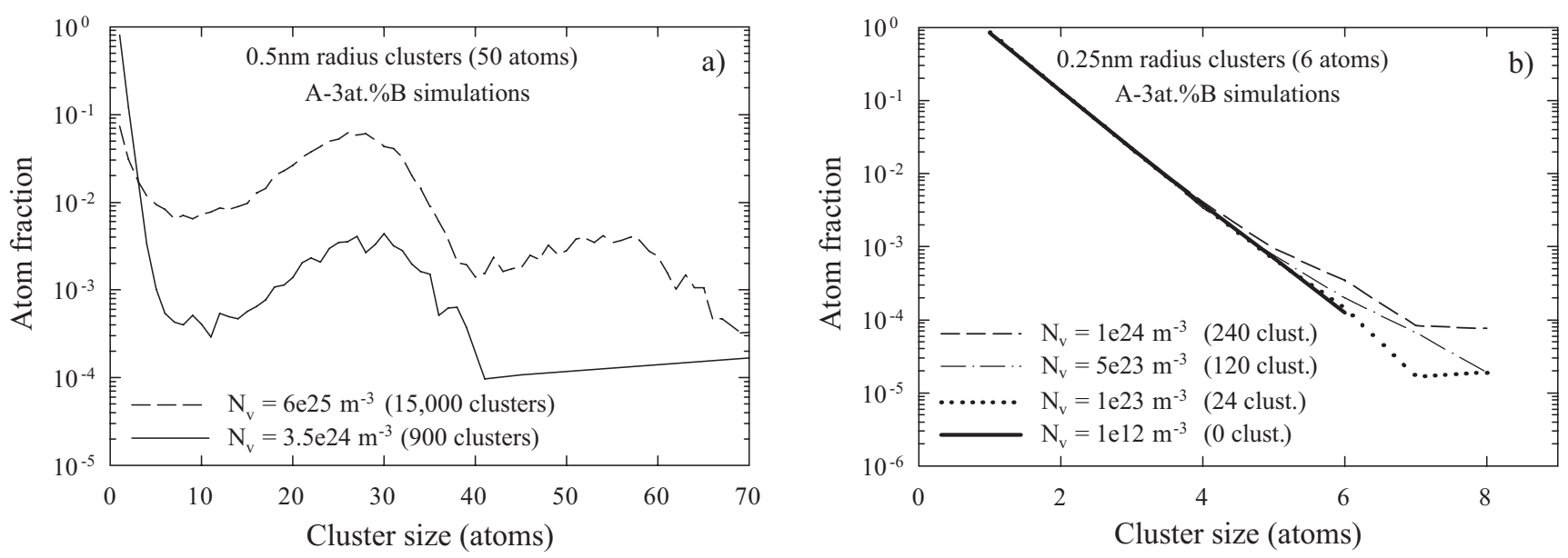

Figure 3: a) Detection of solute clusters of radius $0.5 \mathrm{~nm}$ ( $\sim 50$ atoms), in simulated datasets with an overall solute content of 3 at.\%. b) Detection of solute clusters of radius $0.25 \mathrm{~nm}$ ( $\sim 6$ atoms) at different number densities and the ideal random solute distribution, in simulated datasets with an overall solute content of 3 at.\%.

displacement due to trajectory aberrations [2]. In order to evaluate the ability of this method to detect even the smallest clusters, several datasets were simulated based on binary alloys with 3 at.\% solute concentration (similar to the concentrations contained in the experimental binary alloys). To simulate the additional solute clusters, a series of different solute cluster densities of different sizes were randomly positioned in the volumes. The solute content of the matrix was changed so that the overall composition remained constant. All volumes contain 14 million atoms of which 430,000 are solute atoms in order to ensure statistically reliable results. All simulations were carried out in $3 \mathrm{D}$.

The results observed for simulated datasets with solute clusters which are $0.5 \mathrm{~nm}$ in radius are given in Figure $3 a$. The dashed line shows the situation for which all solutes in the volume are part of solute clusters and, therefore, the cluster density is very high $\left(6 \cdot 10^{25} \mathrm{~m}^{-3}\right.$, which in the given volume results in 15,000 clusters). The continuous line shows the situation where only some solutes are part of solute clusters: the volume contains only 900 clusters, thus only a small percentage of the solute atoms is part of a solute cluster and the rest is randomly distributed in the matrix. It can be seen that both curves display peaks which reach their maximum at $\sim 27$ atoms: this is correct since, due to the $60 \%$ detector efficiency, the actual size of a perfect cluster composed of $\sim 50$ atoms atoms is expected to be $\sim 27$ atoms. The second peak shown in the dashed curve is due to the fact that some clusters will impinge on each other as a result of the high density of clusters.
If solute clusters have a radius of $\sim 0.5 \mathrm{~nm}$, they are large enough to stand out in the analysis. Clusters larger than 10 atoms in size are expected to be observed and, depending on numbers, the results will show a peak corresponding to the correct size of the clusters (taking into account the expected detection efficiency). Difficulties at detecting solute clusters will start to appear when the number of atoms in the cluster is less than 10 atoms. This is because, given a random distribution of solute atoms in an A-3 at.\%B alloy, some solute atoms will be neighbours to the extent that small numbers of naturally occurring clusters up to 7-8 atoms in size are to be expected.

This effect, and the ability of this method to detect extremely small solute clusters is illustrated in Figure 3b. The continuous line shows the natural occurrence of clusters given a random distribution of solute atoms in a binary simulated dataset with a 3 at.\% solute content. Given a large enough statistical sample, it can be demonstrated that the results will lie on a straight line. The other lines show the results from datasets with different number densities of solute clusters which have a $0.25 \mathrm{~nm}$ radius ( $\sim 6$ atoms). Given a 3 at.\% solute content, less than $1 \%$ of the solute atoms in the examples shown in Figure $3 \mathrm{~b}$ are in a cluster configuration, with the rest randomly distributed on the lattice. Nevertheless, this method is able to detect the clustering behaviour as the results deviate from a straight line, indicating that the distribution of solute atoms is no longer random. Furthermore, clusters larger than the one present in 
the random distribution are detected. The results are affected even when as few as 24 clusters $\left(\mathrm{N}_{\mathrm{v}}=1 \cdot 10^{23} \mathrm{~m}^{3}\right)$ which are 6 atoms in size (1.5 unit cells) are present in the volume. At these low detectable number densities, the influence of these small clusters on the mechanical properties will be small when compared to that produced from the random clusters.

In short, the algorithm used is expected to fail only when the solute clusters approach the size and distribution of those occurring naturally due to the solute content of the alloy. In this case, only a minor or negligible influence on the mechanical properties would be expected. These findings provide the confidence that significant clustering behaviour, particularly of the size and distribution suggested by Blavette [3] and Wanderka [4], will be detected if present.

\section{Analysis of binary alloys}

Given that the methodology used is expected to be able to detect even the smallest and most diluted solute clusters, the same technique will now be applied to examine whether solute clusters exist in the binary alloys. Since the alloys have different compositions and, given a random distribution of solutes, the number of randomly clustered atoms changes with solute concentration, each binary alloy was compared to a simulated dataset with exactly the same solute content and similar size. The solute atoms were randomly distributed in the lattice. The comparison will highlight even the slightest deviations from the ideal random distribution of solutes.

The results for $\mathrm{Ni}-\mathrm{X}$ where $\mathrm{X}$ is $\mathrm{Ta}, \mathrm{W}, \mathrm{Pt}$ and $\mathrm{Ir}$ are shown in Figure 4 ( $\mathrm{a}, \mathrm{b}, \mathrm{c}$ and $\mathrm{d}$ respectively). It can be observed that all four alloys follow a near-random distribution of solute atoms: no solute clusters (above the level expected for the random clusters) are detected. Close examination reveals that whilst $\mathrm{W}$ shows a random distribution which matches that of the simulation, Ta displays small numbers of solute clusters in addition to those predicted by the analysis on the simulated dataset. The deviation is a result of two 8-atom clusters which are present in the binary alloy, but are not predicted by the simulations. The data for $\mathrm{Ni}-\mathrm{Ir}$ and $\mathrm{Ni}-\mathrm{Pt}$ also have small deviations from a random distribution, however no 8-atom clusters are detected and the deviations are due to twice the amount of clusters, which are 6 and 7 atoms in size being detected by the algorithm.

The results for Ni-Re are shown in Figure 5a. The experimental and simulated volumes match as far as the solute distribution is concerned. The number of clusters detected by the algorithm strongly suggests that rhenium atoms are randomly distributed in the nickel lattice and their distribution is not biased by any clustering reaction. In other words, no rhenium clusters were found in any of the Ni-Re volumes.

The results strongly suggest that none of the $5 \mathrm{~d}$ transition metals examined in this work exhibits a clustering behaviour in the nickel $f c c$ lattice. Thus, given that the binary alloys have solute content similar to that found in the $\gamma$ phase of commercial alloys, it is likely that rhenium is not prone to clustering in the nickel superalloys. To investigate this further, the same method was applied to examine possible clustering behaviour of Re in the $\gamma$ matrix of the commercial CMSX-4.

Analysis of CMSX-4 alloy

The results from the analysis performed on the $\gamma$ phase in CMSX-4 are shown in Figure 5b. Several volume sections were taken from crept and un-crept samples with only the $\gamma$ phase present in the region being examined using the algorithm. The sections were taken from both very near to the $\gamma / \gamma^{\prime}$ interfaces and the middle of the $\gamma$ channel. The sections taken from the middle of the $\gamma$ channel also have particles of secondary $\gamma^{\prime}$ which precipitate upon cooling. A typical volume displaying primary and secondary $\gamma^{\prime}$ particles is shown in Figure 6a. The volumes were also checked for fluctuations of rhenium content due to microstructural features (i.e. the rhenium enrichment at $\gamma / \gamma^{\prime}$ interfaces). A concentration profile for the $5 \mathrm{~d}$ elements Re, $\mathrm{W}$ and Ta is shown in Figure 6b. The typical local enrichment of Re in the $\gamma$ phase close to the $\gamma / \gamma^{\prime}$ interface is clearly evident. None of the regions of interest showed evidence of rhenium clusters. The data displayed in Figure $5 \mathrm{~b}$ are taken from an area within $10 \mathrm{~nm}$ from a $\gamma / \gamma^{\prime}$ interface, where no secondary $\gamma^{\prime}$ precipitates were present. Previous work by Wanderka et al. [4] suggested that rhenium clusters were present near the interfaces between the $\gamma$ and $\gamma^{\prime}$ phases, where a rhenium enrichment is found. The analysis presented herein strongly suggest that no rhenium clusters are present.

\section{Discussion}

This study calls into question previous work which suggested that discontinuities in ladder diagrams are proof of rhenium clustering. Natural fluctuations in rhenium content in nickel superalloys will occur due to the com- 

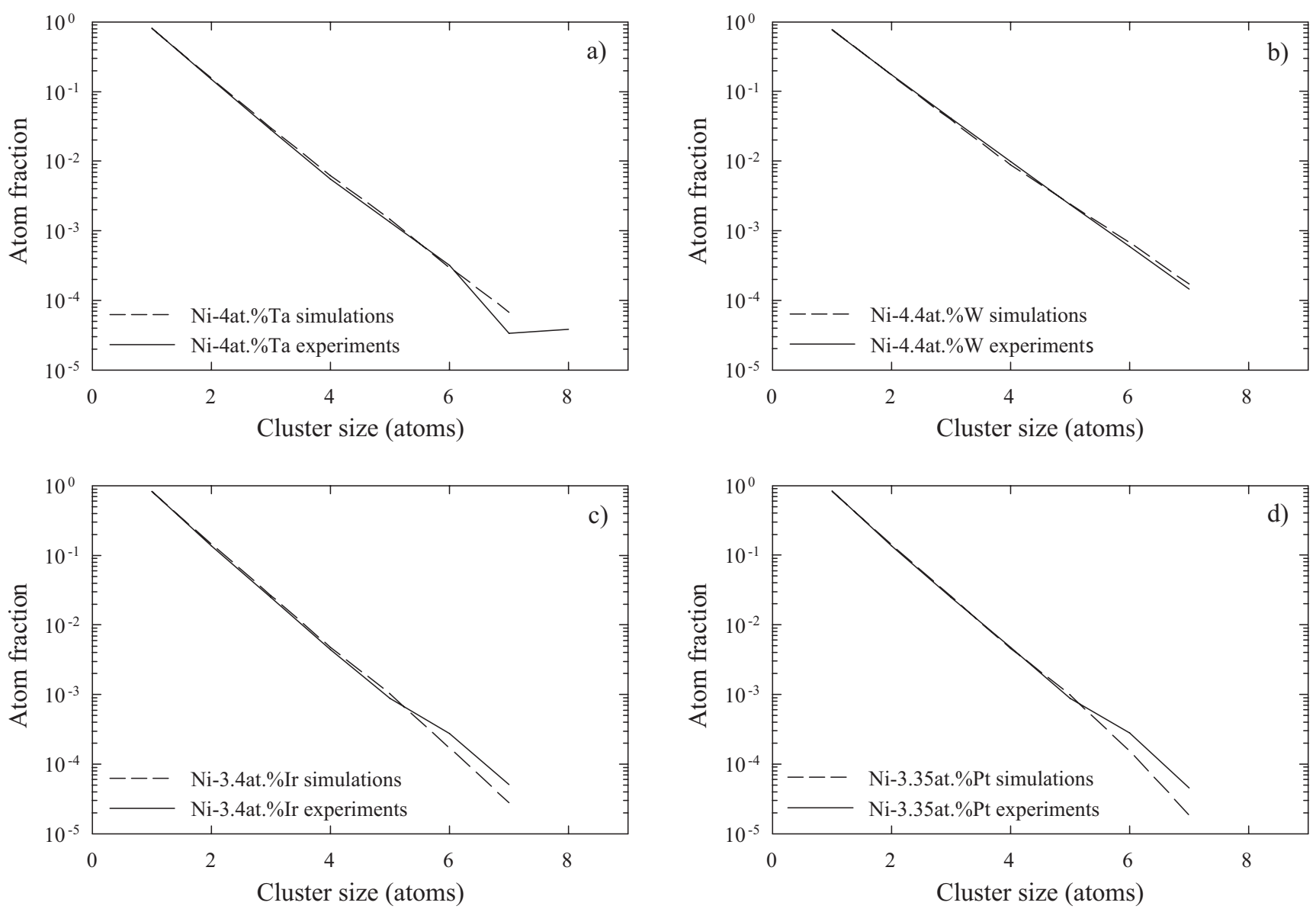

Figure 4: The results obtained when analysing the APT data from the binary alloys a) Ni-Ta, b) Ni-W, c) Ni-Ir and d) Ni-Pt respectively. The results are compared to simulations of equal solute concentration.
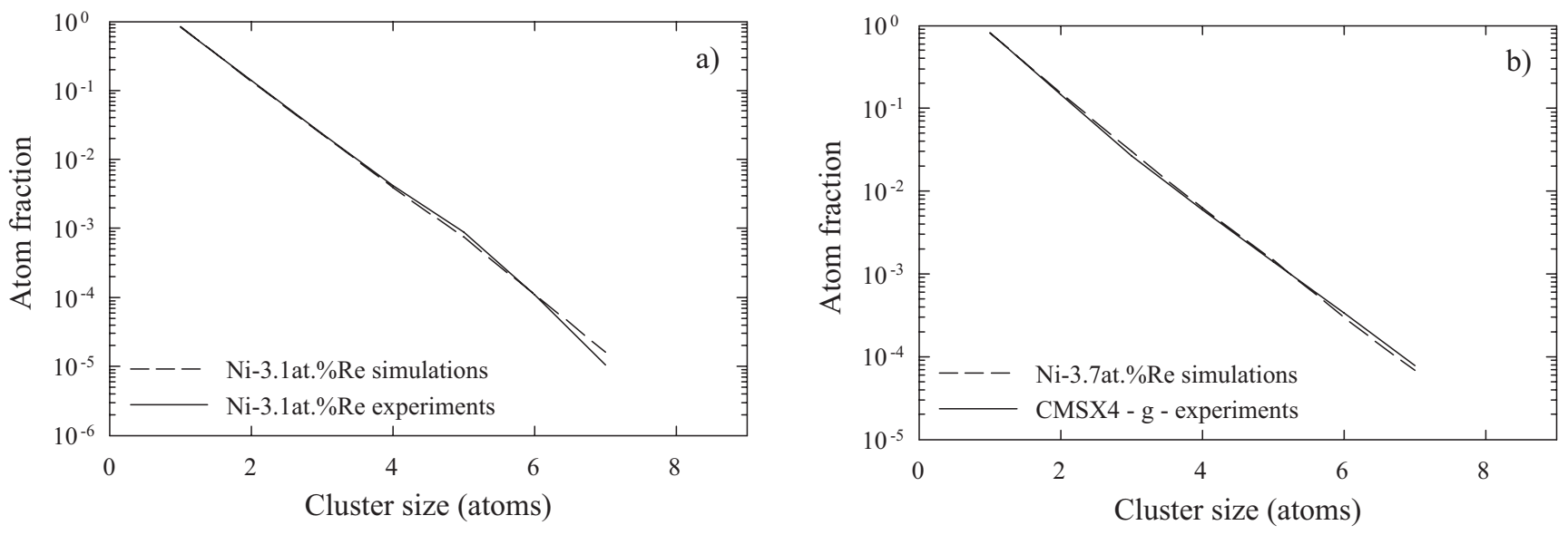

Figure 5: a) The results obtained when analysing the Ni-Re binary alloy compared to a simulated volume with matching solute content which is randomly distributed in the lattice, b) the rhenium distribution found for a dataset taken from the $\gamma$ phase. 

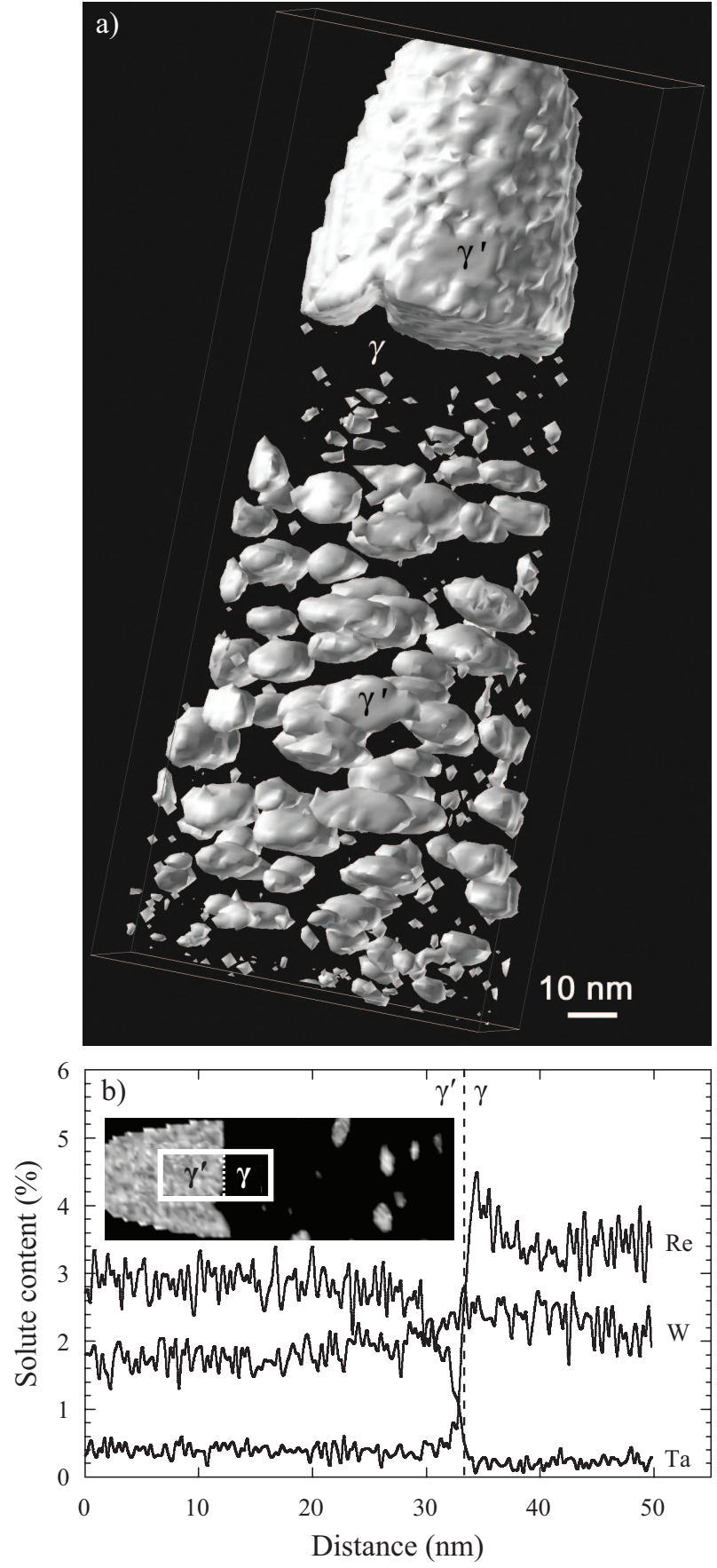

Figure 6: a) The 10 at.\% Al isosurface obtained from a crept CMSX4 sample, showing a small section of a primary $\gamma^{\prime}$ particle, the $\gamma$ channel and several secondary $\gamma^{\prime}$ particles in the $\gamma$ channel. b) The $20 \times 20 \mathrm{~nm}$ concentration profile across the $\gamma / \gamma^{\prime}$ interface shown in a). plicated microstructure and high partitioning coefficient of rhenium. The presence of $\gamma / \gamma^{\prime}$ interfaces, primary $\gamma^{\prime}$, secondary $\gamma^{\prime}$ particles and dendritic segregations will affect the homogeneity of the rhenium distribution in the Ni-based superalloys. Nevertheless the modern 3D atom probe is a viable tool to detect nano-clustering.

The approach of a combined analysis of experimental and simulated atom probe data allows for the investigation of the effect of detection efficiency and atomic displacement on nano-sized solute clusters and to compare these results to those obtained from the experimental datasets.

Modern atom probes are able to collect several millions of atoms: when observing the behaviour of diluted solute atoms, a large statistical sample is required. Nowadays atom probes can deliver the required statistical sizes of the data which can be analysed statistically to yield the distribution of different sizes of clusters. This approach can therefore help to differentiate between the natural occurrence of clusters in an alloy from a real clustering behaviour of the solute atoms in the same alloy.

The analysis was performed on both binary alloys and CMSX-4, since microstructure has an effect on solute atoms distribution. Different sections of the datasets were analysed separately to make sure local enrichments due to $\gamma / \gamma^{\prime}$ interfaces and inhomogeneities due to the high partitioning coefficient of Re between $\gamma$ and $\gamma^{\prime}$ would not affect the results.

The analysis shows that rhenium is randomly distributed in the nickel lattice in a similar way to other elements from the $5 \mathrm{~d}$ series, suggesting that rhenium does not form clusters in the nickel lattice. The same result was obtained when studying the presence of clusters in CMSX-4. Data sections from both in the middle of the $\gamma$ channels and the $\gamma / \gamma^{\prime}$ interfaces were examined to find no evidence of rhenium clusters. It follows that current theories on the interactions between dislocations and rhenium clusters should be revised.

The results obtained in this work imply that a new theory on the mechanisms behind the rhenium effect must be formulated. Single rhenium atoms must act to hinder dislocation motion. This is corroborated by the recent findings by Janotti et al. $[12,13]$ which show that rhenium, amongst the elements from the $5 \mathrm{~d}$ series, is the slowest diffusing element in the nickel $f c c$ lattice. It follows that diffusion mechanisms which control dislocation motion are slower in the presence of rhenium. Further- 
more, rhenium, due to its native $h c p$ crystal structure, will have an effect on the stacking fault energy of nickel. If rhenium decreases the stacking fault energy, partial dislocations will be spaced further apart thereby making it difficult for dislocations to climb.

\section{Conclusions}

The following conclusions can be drawn from this work:

1. When simulating $3 \mathrm{D}$ atom probe datasets, it has been demonstrated that it is important to account for the effect of detection efficiency and positional scattering of the atoms. Additionally, the presence of clusters which occur naturally due to statistical fluctuations needs to be accounted for.

2. It has been shown that $3 \mathrm{D}$ atom probe can detect very small ( $\sim 6$ atoms) clusters at low number densities. However, due to the small sample size, ladder diagrams are not the best method to determine the general behaviour of a solute atom in a host lattice and the possible presence of clusters.

3. No rhenium clusters were detected in Ni-Re, at a rhenium concentration of 3.38 at. $\%$.

4. No solute clusters were detected in any of the Ni-Ta, Ni-W, Ni-Ir and Ni-Pt binary alloys.

5. No rhenium clusters were detected in the $\gamma$ phase of the CMSX-4 superalloy. Analysis was carried out both on crept and un-crept samples and in regions near the $\gamma / \gamma^{\prime}$ interfaces and in the center of the $\gamma$ channels. In all cases, no clusters were detected.

6. It follows that the rhenium-effect cannot be explained by rhenium clusters. New theories for the rhenium-effect are required.

\section{Acknowledgements}

Funding from the United Kingdom's Engineering and Physical Sciences Research Council (EPSRC) under grant $\mathrm{EP} / \mathrm{D} 04619 \mathrm{X} / 1$ is acknowledged (AM and RCR). Research at the Oak Ridge National Laboratory SHaRE User Facility was sponsored by the Scientific User Facilities Division, Office of Science, U.S. Department of Energy (MKM).

\section{References}

[1] R. C. Reed. The Superalloys: Fundamentals and Applications. Cambridge University Press, 2006.

[2] M. K. Miller. Atom Probe Tomography. Kluwer Academic and Plenum Press, New York, 2000.

[3] D. Blavette, P. Caron, and T. Khan. An atom probe investigation of the role of rhenium additions in improving creep resistance of Ni-base superalloys. Scripta Metallurgica, 20(10):1395-1400, 1986.

[4] N. Wanderka and U. Glatzel. Chemical composition measurements of a nickel-base superalloy by atom probe field ion microscopy. Material Science and Engineering A, 203(1-2):69-74, 1995.

[5] P. J. Warren, A. Cerezo, and G. D. W. Smith. An atom probe study of the distribution of rhenium in a nickel-based superalloy. Material Science and Engineering A, 250(1):88-92, 1998.

[6] M. K. Miller and R. C. Reed. Analysis of rhenium clustering in CMSX4 nickel-based superalloys. In J.M. Howe, D.E. Laughlin, J.K. Lee, U. Dahmen, and W.A. Soffa, editors, Proceedings of Solid-Solid Phase Transformation in Inorganic Materials, volume 2, pages 537-542, Point Hilton Resort, Squaw Peak, Phoenix AR, 2005.

[7] K. E. Yoon, D. Isheim, R. D. Noebe, and D. N. Seidman. Nanoscale studies of the chemistry of a Rene N6 superalloy. Interface Science, 9(3-4):249-255, 2001.

[8] K. E. Yoon, R. D. Noebe, and D. N. Seidman. Effects of rhenium addition on the temporal evolution of the nanostructure and chemistry of a model NiCr-Al superalloy. I: Experimental observations. Acta Materialia, 55(4):1145-1157, 2007.

[9] K. E. Yoon, R. D. Noebe, and D. N. Seidman. Effects of rhenium addition on the temporal evolution of the nanostructure and chemistry of a model Ni-CrAl superalloy. II: Analysis of the coarsening behavior. Acta Materialia, 55(4):1159-1169, 2007.

[10] D. Blavette, E. Cadel, C. Pareige, B. Deconihout, and P. Caron. Phase transformation and segregation to lattice defects in Ni-base superalloys. Microscopy and Microanalysis, 13(6):464-483, 2007.

[11] J. Rüsing, N. Wanderka, U. Czubayko, V. Naundorf, D. Mukherji, and J. Rosler. Rhenium distribution in the matrix and near the particle-matrix interface in 
a model Ni-Al-Ta-Re superalloy. Scripta Materialia, 46(3):234-240, 2002.

[12] A. Janotti, M. Krcmar, C. L. Fu, and R. C. Reed. Solute diffusion in metals: Larger atoms can move faster. Physical Review Letters, 92(8):085901, 2004.

[13] C. L. Fu, R. C. Reed, A. Janotti, and M. Krcmar. On the diffusion of the solutes in the superalloys. In K.A. Green, T.M. Pollock, H. Harada, T.E. Howson, R.C. Reed, J.J. Schirra, and S. Walston, editors, Superalloys 2004, pages 189-195. The Minerals, Metals and Materials Society, Warrendale, PA, USA, 2004.

[14] B. Ravel. A practical introduction to multiple scattering theory. Journal of Alloys and Compounds, 401(1-2):118-126, 2005.

[15] A. Mottura, R. T. Wu, M. W. Finnis, and R. C. Reed. A critique of rhenium clustering in Ni-Re alloys using extended X-ray absorption spectroscopy. Acta Materialia, in print, 2008.

[16] T. F. Kelly and M. K. Miller. Atom probe tomography. Review of Scientific Instruments, 78(2):031101, 2007.

[17] R. C. Reed, N. Matan, D. C. Cox, M. A. Rist, and C. M. F. Rae. Creep of CMSX4 superalloy single crystals: effects of rafting at high temperature. Acta Materialia, 47(12):3367-3381, 1999.

[18] Alloy Phase Diagrams, volume 3 of ASM Handbook. 1992.

[19] M. K. Miller and J. M. Hyde. An atom probe simulator. In 11th European Congress on Electron Microscopy, Dublin, Ireland, 1996. 